\title{
Stabiliser Fault Emergency Control using Reconguration to Preserve Power System
} Stability

Pedersen, Andreas Søndergaard; Richter, Jan H.; Tabatabaeipour, Mojtaba; Jóhannsson, Hjörtur; Blanke, Mogens

\section{Published in:}

Proceedings of the 19th IFAC World Congress

Publication date:

2014

Link back to DTU Orbit

Citation (APA):

Pedersen, A. S., Richter, J. H., Tabatabaeipour, M., Jóhannsson, H., \& Blanke, M. (2014). Stabiliser Fault Emergency Control using Reconguration to Preserve Power System Stability. In Proceedings of the 19th IFAC World Congress International Federation of Automatic Control. I F A C Workshop Series Vol. 19 No. 1

\section{General rights}

Copyright and moral rights for the publications made accessible in the public portal are retained by the authors and/or other copyright owners and it is a condition of accessing publications that users recognise and abide by the legal requirements associated with these rights.

- Users may download and print one copy of any publication from the public portal for the purpose of private study or research.

- You may not further distribute the material or use it for any profit-making activity or commercial gain

- You may freely distribute the URL identifying the publication in the public portal 


\title{
Stabiliser Fault Emergency Control using Reconfiguration to Preserve Power System Stability
}

\author{
Andreas S. Pedersen ${ }^{*, * * *}$ Jan H. Richter ${ }^{* * * *}$ \\ Mojtaba Tabatabaeipour ${ }^{*, * * *}$ Hjörtur Jóhannsson ${ }^{* *, * * *}$ \\ Mogens Blanke*,*** \\ * Automation and Control Group at Dept. of Electrical Engineering; \\ emails: $\{$ asped,setaba,mb@elektro.dtu.dk\} \\ ** Centre for Electric Power and Energy at Dept. of Electrical \\ Engineering; email: hjjo@elektro.dtu.dk \\ *** Technical University of Denmark, 2800 Kgs. Lyngby, Denmark \\ **** Siemens AG, Industry Sector, Gleiwitzer Str. 555, 90475 \\ Nürnberg, Germany; email: janrichter@siemens.com
}

\begin{abstract}
Stabiliser faults in multi-machine power systems are examined in this paper where fault-masking and system reconfiguration of the nonlinear system is obtained using a virtual actuator approach. Phasor Measurement Units, which can be integrated in wide-area transmission grids to improve the performance of power system stabilisers, are utilised when reconfiguring remaining stabilisers after one has been inoperable by a local failure. A stabilitypreserving reconfiguration is designed using absolute stability results for Lure type systems: The calculation of the virtual actuator that relies on a solution of a linear matrix inequality (LMI) is detailed in the paper. Simulation results of a benchmark transmission system show the ability of the fault-tolerant reconfiguration strategy to maintain wide-area stability of a power system despite failure in a stabiliser.
\end{abstract}

Keywords: Power-system stabilisers; Fault-tolerant systems; System failure and recovery; Absolute stability.

\section{INTRODUCTION}

Multi-machine power systems can experience problems related to low-frequency oscillations (in the 0.1-2 $\mathrm{Hz}$ range). These oscillations arise from the power and phase-angle relationship interacting with generators' inertia, forming an equivalent multi-mass-spring system. Large scale power systems exhibit both local and inter-area oscillations. Local oscillations are related to oscillation of a single machine with respect to the rest of the system and inter-area oscillations are related to oscillations of a group of plants against another group. These problems are intensified in highly stressed conditions such as emergency conditions. If these oscillations are poorly damped, they might lead to a loss of synchronism between synchronous machines and cause cascading tripping events.

Power system stabilisers (PSSs) are effective tools to damp such low-frequency oscillations. They are added on voltage controlling elements of the power system and adds auxiliary signals to the voltage regulation, compensating oscillations in active power transmission [Kundur, 1994]. The performance of a power system is usually analysed by checking the eigenproperties, and improved by adding active damping control to the electromechanical modes.

\footnotetext{
‡ This work was supported through the SOSPO project by the Danish Council for Strategic Research under grant no. 11-116794
}

The performance of locally designed PSSs can be improved using wide-area measurement signals and wide-area control (WAC) (as shown by Snyder et al. [1998]). With the growing use of new technologies such as phasor measurement units (PMU) and fast communication technologies, WAC have given new possibilities in power system operation. This includes use of such wide area information to improve stability and for emergency control [Begovic et al., 2005]. When the PSSs in a multi-machine power system work collaboratively, a proper functionality is expected from each individual stabiliser as a fault in one stabiliser could cause unsatisfactory performance or even instability of the collective control. In the present systems, cascaded tripping is a concern if an individual PSS fails. In this paper, we show how we can use wide-area measurement signals and design a wide-area reconfiguration block that can reconfigure the control action and stabilise the system in an event of failure in some of the local stabilisers.

The purpose of reconfiguring the control after a fault is to preserve specific properties of the closed-loop system [Blanke et al., 2006]. In this work we use the virtual actuator method for reconfiguration [Richter, 2011b]. The idea of virtual actuator is to keep the nominal controller in the loop and transform the input signals designed for the nominal plant to signals appropriate for the remaining healthy actuators. The reconfiguration method is applied to power systems with PSSs installed on synchronous 
generators. When a PSS fails, a wide-area virtual actuator is designed that restructures the nominal control loop by using the remaining healthy PSSs to compensate the active damping that is missing due to the fault. The advantage of this approach is to obtain fault-tolerance that is transparent to other controls. Nominal design and tuning can be used for the remaining stabilisers, faulttolerance is obtained through a reconfiguration block.

Design of wide-area stabilisers was pursued in [Snyder et al., 1998], where locale controls were extended with remote measurements to improve observability of interarea modes. In [Kamwa et al., 2001], wide-area information was used in a hierarchical control scheme. A level of fault tolerance was obtained in (Chen et al. [2006], Chen and Guo [2005]) where a robust wide-area controller used mixed $\mathcal{H}_{2} / \mathcal{H}_{\infty}$ output-feedback control. Adaptive stabilisers using wide-area information were designed in [Zhang et al., 1993] and [Ni et al., 2000]. The test example in [Ni et al., 2000] showing fault tolerance after a PSS failure will also be used in this paper.

The contributions of this work are the following: A widearea fault-tolerant virtual actuator is designed for the power system which stabilises the system after a fault in local stabilisers. The proposed method does not require changes in the local controller but accommodates faults by adding signals to the output of them. The nonlinearities in the model of the system are taken into account by modelling the power system in the Lure form. A new design method for virtual actuator for Lure systems based on absolute stability theory is proposed which improves the results of Richter et al. [2012].

The paper is organised as follows. The dynamic power system model used for stabiliser design is first described and instability mechanisms are explained. The nonlinear nature of the emergency dynamics is then discussed and a Lure form is introduced to enable generic analysis. Section 3 then discusses reconfiguration based on a virtual actuator approach for nonlinear systems and extends virtual actuator based theory to cope at ease with the problem at hand. A benchmark test system is presented in section 4 that develops instability when one of the power system stabiliser units fail and simulations are performed showing successful reconfiguration and regain of stability using the new approach.

\section{POWER SYSTEM MODEL}

The flux-decay model of a generator with an automatic voltage regulator (AVR) is considered as this model is often used for stabiliser design. In the flux-decay model, the ammortisseur effects are neglected. The dynamics of machine $i$ in the network is (from [Sauer and Pai, 1998]),

$$
\begin{aligned}
\dot{\delta}_{i}^{\prime}= & \omega_{0} \omega_{i} \\
M_{i} \dot{\omega}_{i}= & P_{m, i}-P_{e, i}-D_{i} \omega_{i} \\
T_{d 0, i}^{\prime} \dot{E}_{q, i}^{\prime}= & -\left(1-\left(x_{d}-x_{d}^{\prime}\right) B_{i i}\right) E_{q, i}^{\prime}+\left(x_{d}-x_{d}^{\prime}\right) i_{d} \\
& \quad+K_{A}\left(E_{A V R, i}-v_{\mathrm{ref}}+v_{p s s}\right) \\
T_{R, i} \dot{E}_{A V R, i}= & -E_{A V R, i}+E_{t}
\end{aligned}
$$

where, for each generator $i$

$P_{m, i} \quad$ Mechanical input
$P_{e, i} \quad$ Electrical output

$E_{A V R, i} \quad$ AVR filter

$D_{i} \quad$ Damping power coefficient

$x_{d}, x_{q}, x_{d}^{\prime} d, q$-axis synchronous, transient reactances

$i_{d}, i_{q} \quad d, q$-axis current

$v_{\text {ref }} \quad$ Reference terminal voltage

$v_{\text {pss }} \quad$ Stabiliser input

$T_{R, i} \quad$ AVR time constant

$T_{d 0, i}^{\prime} \quad d$-axis transient open circuit time constant

$E_{i}^{\prime} \angle \delta_{i} \quad$ Internal potential

$E_{q, i}^{\prime} \angle \delta_{i}^{\prime} \quad q$-axis component of the internal potential

$\omega_{i} \quad$ Rotor speed devation from a reference

$\omega_{0} \quad$ Synchronous speed

$K_{A} \quad$ AVR gain

$E_{t} \quad$ Terminal voltage

The stator equations are

$$
\begin{aligned}
& E_{t} \sin \left(\delta_{i}-\delta_{t, i}\right)-x_{q} i_{q}=0 \\
& E_{t} \cos \left(\delta_{i}-\delta_{t, i}\right)-x_{d}^{\prime} i_{d}+E_{q, i}^{\prime}=0 .
\end{aligned}
$$

The network equations between the generators needs to follow the current-balance $\mathbf{i}=\mathbf{Y} \mathbf{v}$, where $\mathbf{v}$ is the vector of bus voltage, $\mathbf{i}$ is the bus currents and $\mathbf{Y}$ the admittance matrix. Using the Kron-reduced internal node network, the power and currents can be calculated using

$$
\begin{aligned}
P_{e, i} & =E_{i}^{\prime 2} G_{i i}+E_{i}^{\prime} \sum_{j \neq i} E_{j}^{\prime} Y_{i j} \sin \left(\delta_{i}-\delta_{j}+\alpha_{i j}\right) \\
i_{d, i} & =-E_{i}^{\prime} B_{i i}+\sum_{j \neq i} E_{j}^{\prime} Y_{i j} \cos \left(\delta_{i}-\delta_{j}+\alpha_{i j}\right) \\
i_{q, i} & =E_{i}^{\prime} G_{i i}+\sum_{j \neq i} E_{j}^{\prime} Y_{i j} \sin \left(\delta_{i}-\delta_{j}+\alpha_{i j}\right),
\end{aligned}
$$

where $\tilde{Y}_{i j}=Y_{i j} \angle \phi_{i j}=G_{i j}+j B_{i j}$ is the $i j^{\text {th }}$ element of $\mathbf{Y}$ and $\alpha_{i j}=\arctan \frac{G_{i j}}{B_{i j}}$. The model is formulated as in [Kakimoto et al., 1980], where it was assumed that each internal voltage lags behind the $q$-axis of each generator by a constant angle. As each machine has four states, an n-machine network would comprise $4 n$ states.

The power system model can obtain oscillatory behaviour under certain circumstances related to the transmission line properties between machines, the level of power transmitted and to the control system parameters. gg behaviour is encountered under conditions of high reactance of the system (transmission and consumers) and high generator outputs. High synchronizing torque is then needed for generators, but the associated high gain in automatic voltage regulation loops cause deteriorated system damping [Kundur, 1994]. Additional damping is achieved through adding a stabilizing loop to generator control through the auxiliary input $v_{p s s}$. This power system stabiliser (PSS) loop obtains damping by controlling generator torque as a function of deviation of rotational speed from its nominal value. The design of PSS controllers is commonly done using a linearised version of the system equations. In high load conditions or in emergencies, conditions move to the nonlinear parts of the envelope of system dynamics. When we wish to obtain guarantees for stability during emergency situations, and associated large transients, the normal approach of linear design of stabilisers will not suffice. Instead a nonlinear model and an adequate nonlinear design approach are required. 


\subsection{Lure System}

Aiming at analysis of properties of the nonlinear system Eqs. 1 to 8, and subsequent design of a stabilizing control, the system is conveniently described in generic terms using a Lure form as system representation. With active power and the $d$-axis current of each machine as nonlinear internal feedback in the model, see [Kakimoto et al., 1980], the general Lure system formulation is,

$$
\Sigma_{P}:\left\{\begin{array}{l}
\dot{\boldsymbol{x}}(t)=\boldsymbol{A} \boldsymbol{x}(t)+\boldsymbol{B}_{v} \boldsymbol{v}(t)+\boldsymbol{B u}_{c}(t)+\boldsymbol{B}_{\boldsymbol{d}} \boldsymbol{d}(t) \\
\boldsymbol{v}(t)=\boldsymbol{\varphi}\left(\boldsymbol{C}_{v} \boldsymbol{x}(t)\right) \\
\boldsymbol{y}(t)=\boldsymbol{C} \boldsymbol{x}(t) \\
\boldsymbol{z}(t)=\boldsymbol{C}_{\boldsymbol{z}} \boldsymbol{x}(t)
\end{array}\right.
$$

The feedback signal $\boldsymbol{v}$ is obtained using the nonlinear characteristic $\varphi(\cdot): \mathbb{R}^{s} \mapsto \mathbb{R}^{s}$ satisfying the following assumption.

Assumption 1. (Nominal Lure nonlinearity). The function $\boldsymbol{\varphi}$ is decomposed, element-wise Lipschitz, and sectorbounded in the sector $[0, \boldsymbol{K}]$, with $\boldsymbol{K}=\operatorname{diag}\left(k_{1}, \ldots, k_{s}\right)$.

It is convenient to transform the sector condition $\left[-\alpha_{i}, \alpha_{i}\right]$ to $[0,1]$. This is obtained with a loop transformation $\boldsymbol{A}^{\prime}=$ $\boldsymbol{A}-\boldsymbol{B} \boldsymbol{\Lambda} \boldsymbol{C}, \quad \boldsymbol{B}^{\prime}=2 \boldsymbol{B} \boldsymbol{\Lambda}$ where $\boldsymbol{\Lambda}=\operatorname{diag}\left(\alpha_{1}, \alpha_{2}, . ., \alpha_{n}\right)$ is used. In the power system model, the Lure nonlinearity consists of the output power and the $d$-axis current for each machine. To bound the nonlinearity, the following assumption is made.

Assumption 2. (State bounds). It is assumed that the quadrature axis internal voltage satisfies $\left|E_{q, i}^{\prime}-\overline{E_{q, i}^{\prime}}\right| \leq$ $E_{\Delta}$, where $\overline{E_{q, i}^{\prime}}$ is the nominal voltage.

This assumption puts a bound on the Lure nonlinearity. If the deviation $E_{\Delta}$ is chosen appropriatly, it should not affect the result.

The Lure system (8) is controlled by means of some given nominal controller $\Sigma_{C}$. Power System Stabilisers control strategies usually involves using the generators angular frequency or the terminal frequency deviation in a supplementary feedback block through $v_{p s s}$. The following assumption is made on the nominal closed-loop system.

Assumption 3. (Nominal closed-loop stability). The given nominal closed-loop system of $\Sigma_{P}$ and $\Sigma_{C}$ is input-to-state stable $^{1}$ w.r.t. the inputs $(\boldsymbol{r}, \boldsymbol{d})$.

Faults change the nominal Lure system (8) to the faulty Lure system

$\Sigma_{P f}:\left\{\begin{array}{l}\dot{\boldsymbol{x}}_{f}(t)=\boldsymbol{A}_{f} \boldsymbol{x}_{f}(t)+\boldsymbol{B}_{v} \boldsymbol{v}_{f}(t)+\boldsymbol{B}_{f} \boldsymbol{u}_{f}(t)+\boldsymbol{B}_{\boldsymbol{d}} \boldsymbol{d}(t) \\ \boldsymbol{v}_{f}(t)=\boldsymbol{\varphi}_{f}\left(\boldsymbol{C}_{v} \boldsymbol{x}_{f}(t)\right) \\ \boldsymbol{y}_{f}(t)=\boldsymbol{C} \boldsymbol{x}_{f}(t) \\ \boldsymbol{z}_{f}(t)=\boldsymbol{C}_{\boldsymbol{z}} \boldsymbol{x}_{f}(t),\end{array}\right.$

To distinguish the faulty system behavior from the nominal behavior, all signals that are affected by faults are labeled by subscript $f$. The pair $\left(\boldsymbol{A}_{f}, \boldsymbol{B}_{f}\right)$ is assumed to be stabilizable. A PSS failure is an event that changes the

1 A system is ISS if there exists functions $\beta \in \mathcal{K} \mathcal{L}, \gamma \in \mathcal{K}_{\infty}$ such that $|x(t)| \leq \beta\left(\left|x^{0}\right|, t\right)+\gamma\left(\|u\|_{\infty}\right)$ nominal input matrix $\boldsymbol{B}$ to the faulty input matrix $\boldsymbol{B}_{f}$ by setting the corresponding row to zero.

\section{LURE VIRTUAL ACTUATOR FOR CONTROL RECONFIGURATION}

In this section we will present a new reconfiguration result using a passivity-based stabilizing design of Lure type systems extending the result from Richter et al. [2012].

The concept of fault-hiding using control reconfiguration is shown in Figure 1. After a fault, the controller $\Sigma_{C}$ interconnected to the faulty plant by means of the connections $y_{c}=y_{f}$ and $u_{c}=u_{f}$ is generally not suitable for controlling the faulty system. In particular, in the case of stabiliser failures, the loop is partially opened. The reconfiguration block $\Sigma_{R}$ will hide the system fault from the controller, and regain stability of the closed-loop. The

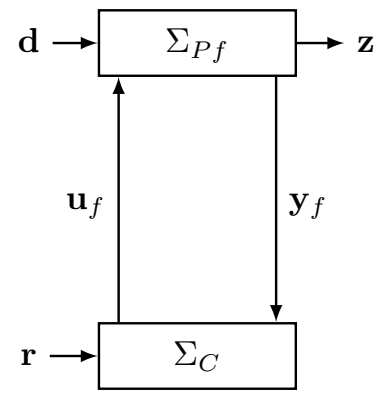

(a) Controller on faulty plant

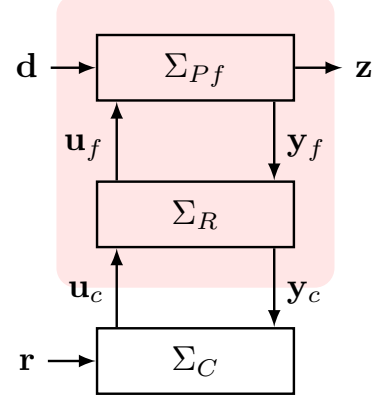

(b) Reconfiguration block hides fault
Fig. 1. Illustration of fault hiding. The reconfiguration restructures the nominal control and modifies the output, to hide the fault from the controller.

virtual actuator implementation is shown in Figure 2. The reconfiguration block $\Sigma_{R}$ proposed in this paper is a Lure virtual actuator

$$
\Sigma_{A}:\left\{\begin{aligned}
& \dot{\boldsymbol{x}}_{\Delta}(t)= \boldsymbol{A}_{\Delta} \boldsymbol{x}_{\Delta}(t)+\left(\boldsymbol{A}-\boldsymbol{A}_{f}\right) \boldsymbol{x}_{f}(t)+\boldsymbol{B}_{v} \boldsymbol{v}_{\Delta}(t) \\
&+\boldsymbol{B}_{\Delta} \boldsymbol{u}_{c}(t) \\
& \boldsymbol{x}_{\Delta}(0)= \boldsymbol{x}_{\Delta 0} \\
& \boldsymbol{v}_{\Delta}(t)=\boldsymbol{\varphi}\left(\boldsymbol{C}_{v}\left(\boldsymbol{x}_{\Delta}(t)+\boldsymbol{x}_{f}(t)\right)\right)-\boldsymbol{\varphi}_{f}\left(\boldsymbol{C}_{v} \boldsymbol{x}_{f}(t)\right) \\
& \boldsymbol{u}_{f}(t)=\boldsymbol{M} \boldsymbol{x}_{\Delta}(t)+\boldsymbol{N} \boldsymbol{u}_{c}(t) \\
& \boldsymbol{y}_{c}(t)=\boldsymbol{y}_{f}(t)+\boldsymbol{C} \boldsymbol{x}_{\Delta}(t) \\
& \boldsymbol{A}_{\Delta} \triangleq \boldsymbol{A}-\boldsymbol{B}_{f} \boldsymbol{M}, \boldsymbol{B}_{\Delta} \triangleq \boldsymbol{B}-\boldsymbol{B}_{f} \boldsymbol{N}
\end{aligned}\right.
$$

(Fig. 1, $\Sigma_{R}=\Sigma_{A}$ ). The virtual actuator $\Sigma_{A}$, whose linear form was introduced in [Steffen, 2005], expresses the difference between nominal and reconfigured dynamics in its state $\boldsymbol{x}_{\Delta}$ and tries to keep this difference small. The matrices $\boldsymbol{M}$ and $\boldsymbol{N}$ are free design parameters that may be used to affect the virtual actuator behavior. Note that the implementation of the Lure virtual actuator requires the knowledge of the state $\boldsymbol{x}_{f}$ of the faulty Lure system, which must either be measured or estimated using an observer ${ }^{2}$.

Although we are primarily interested in actuator failures (i.e. PSS failures), we define more general actuator faults.

\footnotetext{
2 The preservation of stability after introducing an observer is expected but must be analysed separately; a generic discussion of the combination of nonlinear virtual actuators with nonlinear observers
} is available in [Richter, 2011a]. 
The method presented below are applicable to the following definition of faults.

Definition 1. (Actuator and internal faults). An actuator fault $f$ is an event that changes the nominal input matrix $\boldsymbol{B} \in \mathbb{R}^{(n \times m)}$ to the faulty input matrix $\boldsymbol{B}_{f} \in \mathbb{R}^{(n \times m)}$ of the same dimensions. An internal fault is an event that changes the system matrix $\boldsymbol{A}$ to $\boldsymbol{A}_{f}$ and the nominal characteristic $\varphi: \mathbb{R}^{s} \mapsto \mathbb{R}^{s}$ to the faulty characteristic $\varphi_{f}: \mathbb{R}^{s} \mapsto \mathbb{R}^{s}$ of identical dimension.

In this paper, we assume that faults appear abruptly and remain effective once they have occurred.

\subsection{Fault-hiding property and separation principle}

In order to prove the strict fault-hiding constraint, the state transformation $\boldsymbol{x}_{f}(t) \rightarrow \tilde{\boldsymbol{x}}(t) \triangleq \boldsymbol{x}_{f}(t)+\boldsymbol{x}_{\Delta}(t)$ is applied, after which the reconfigured plant (9), (10) is described by

$$
\begin{aligned}
\left(\begin{array}{c}
\dot{\tilde{\boldsymbol{x}}}(t) \\
\dot{\boldsymbol{x}}_{\Delta}(t)
\end{array}\right)= & \left(\begin{array}{cc}
\boldsymbol{A} & \mathbf{0} \\
\mathbf{0} & \boldsymbol{A}_{\Delta}
\end{array}\right)\left(\begin{array}{c}
\tilde{\boldsymbol{x}}(t) \\
\boldsymbol{x}_{\Delta}(t)
\end{array}\right)+\left(\begin{array}{c}
\boldsymbol{B} \\
\boldsymbol{B}_{\Delta}
\end{array}\right) \boldsymbol{u}_{c}(t) \\
& +\left(\begin{array}{c}
\boldsymbol{B}_{v} \tilde{\boldsymbol{v}}(t) \\
\boldsymbol{B}_{v} \boldsymbol{v}_{\Delta}(t)
\end{array}\right)+\left(\begin{array}{c}
\boldsymbol{B}_{\boldsymbol{d}} \\
\mathbf{0}
\end{array}\right) \boldsymbol{d}(t) \\
\tilde{\boldsymbol{x}}(0)= & \boldsymbol{x}_{0}+\boldsymbol{x}_{\Delta 0}, \boldsymbol{x}_{\Delta}(0)=\boldsymbol{x}_{\Delta 0} \\
\tilde{\boldsymbol{v}}(t)= & \boldsymbol{\varphi}\left(\boldsymbol{C}_{v} \tilde{\boldsymbol{x}}(t)\right) \\
\boldsymbol{v}_{\Delta}(t)= & \boldsymbol{\varphi}\left(\boldsymbol{C}_{v} \tilde{\boldsymbol{x}}(t)\right)-\boldsymbol{\varphi}_{f}\left(\boldsymbol{C}_{v}\left(\tilde{\boldsymbol{x}}(t)-\boldsymbol{x}_{\Delta}(t)\right)\right) \\
\boldsymbol{y}_{c}(t)= & \boldsymbol{C} \tilde{\boldsymbol{x}}(t), \boldsymbol{z}_{f}(t)=\boldsymbol{C}_{\boldsymbol{z}} \tilde{\boldsymbol{x}}(t)-\boldsymbol{C}_{\boldsymbol{z}} \boldsymbol{x}_{\Delta}(t) .
\end{aligned}
$$

This model shows that $\boldsymbol{y}_{c}$, the measured output made available to the controller, depends only on the state $\tilde{\boldsymbol{x}}$, which is governed by the nominal dynamics denoted by

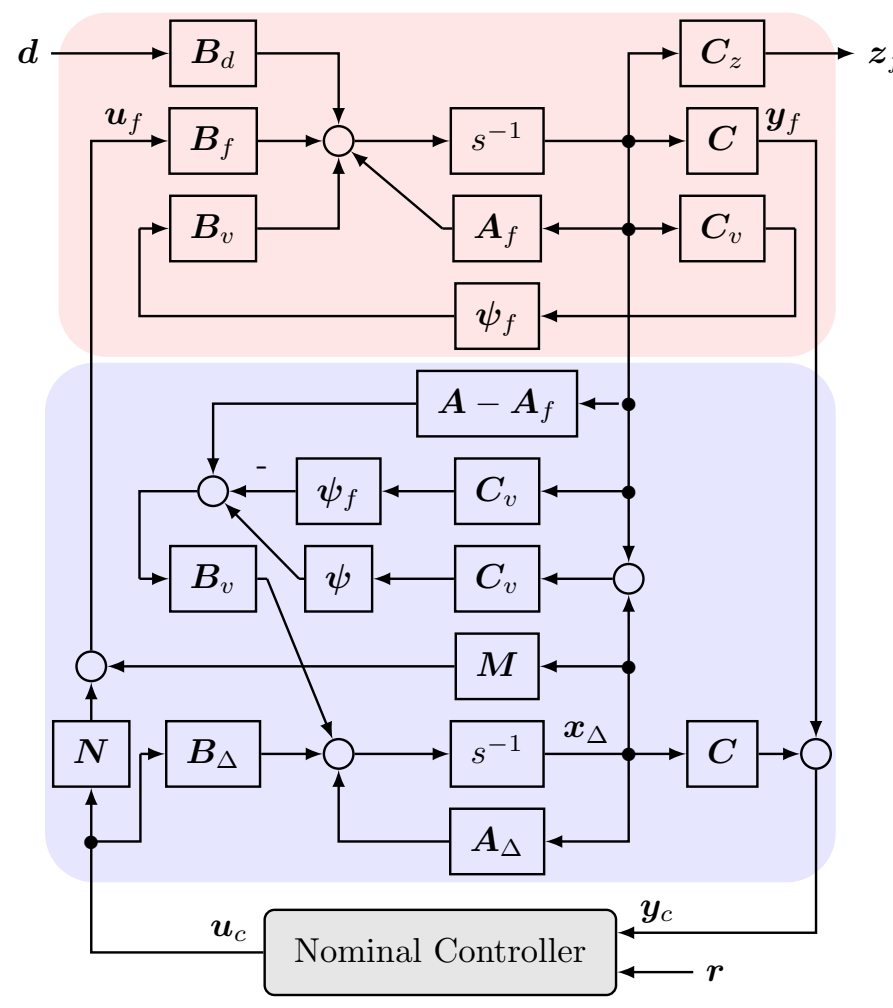

Fig. 2. Virtual actuator implemention for Lure-type systems.
$\boldsymbol{x}_{\Delta 0}=\mathbf{0}$, which proves that the Lure virtual actuator satisfies the strict fault-hiding constraint. Due to Assumption 3 , the interconnection $\left(\Sigma_{\tilde{P}}, \Sigma_{C}\right)$ is ISS.

The difference system is, however, affected by the dynamics of the state variable $\tilde{\boldsymbol{x}}$ through the variable $\boldsymbol{v}_{\Delta}$ (but not the converse, which would contradict fault hiding). The nominal closed-loop system $\left(\Sigma_{\tilde{P}}, \Sigma_{C}\right)$ is connected in series to the difference system $\Sigma_{A}$, which implies that the series interconnection theorem for input-to-state stable systems is applicable, where the first system $\Sigma_{\tilde{P}}$ is ISS by Assumption 3 and it remains to ensure by proper design that the difference system is ISS w.r.t. the inputs $\boldsymbol{u}_{c}(t)$ and $\tilde{\boldsymbol{x}}(t)$.

\subsection{Passivity-based stability recovery}

It remains to give sufficient conditions for input-to-state stability of the difference system $\Sigma_{A}$ w.r.t. its external inputs.

Theorem 1. (Global reconfigured closed-loop ISS).

Consider the faulty Lure system (9) under Assumptions 3, 1 , and let $\boldsymbol{S}=\boldsymbol{K}_{f}^{-1}$. The reconfigured closed-loop system is globally ISS if there exists $\boldsymbol{X}=\boldsymbol{X}^{T} \succ 0$ and $\boldsymbol{Y}$ such that the matrix inequality

$$
\left(\begin{array}{cc}
-\left(\boldsymbol{X} \boldsymbol{A}^{T}+\boldsymbol{A} \boldsymbol{X}-\boldsymbol{B}_{f} \boldsymbol{Y}-\boldsymbol{Y}^{T} \boldsymbol{B}_{f}^{T}\right)-\boldsymbol{X} \boldsymbol{C}_{v}^{T}-\boldsymbol{B}_{v} \\
\star & \boldsymbol{S}+\boldsymbol{S}^{T}
\end{array}\right) \succ 0
$$

is satisfied, where $\boldsymbol{M}=\boldsymbol{Y} \boldsymbol{X}^{-1}$.

Proof. We first consider the unforced difference system (for $\boldsymbol{u}_{c}=\mathbf{0}, \tilde{\boldsymbol{x}}=\mathbf{0}$ ) and show that satisfaction of LMI (12) implies global asymptotic stability of the difference system. According to the circle criterion, the unforced difference system is absolutely stable at the origin if its linear subsystem is passive, which is the case according to Boyd et al. [1994] if the matrix inequality

$$
\left(\begin{array}{cc}
-\left(\boldsymbol{A}-\boldsymbol{B}_{f} \boldsymbol{M}\right)^{T} \boldsymbol{P}-\boldsymbol{P}\left(\boldsymbol{A}-\boldsymbol{B}_{f} \boldsymbol{M}\right) & -\boldsymbol{C}_{v}^{T}-\boldsymbol{P} \boldsymbol{B}_{v} \\
\star & \boldsymbol{S}+\boldsymbol{S}^{T}
\end{array}\right) \succ 0
$$

is feasible in the variables $\boldsymbol{P}=\boldsymbol{P}^{T} \succ 0$ and $\boldsymbol{M}$. The latter inequality is nonlinear for the purpose of designing $\boldsymbol{M}$ due to products between variables $\boldsymbol{P}$ and $\boldsymbol{M}$. The following standard trick turns it into an equivalent LMI: the Schur lemma turns it into the equivalent inequalities $\boldsymbol{S}+\boldsymbol{S}^{T} \succ 0$ and $-\left(\boldsymbol{A}^{T} \boldsymbol{P}+\boldsymbol{P} \boldsymbol{A}-\boldsymbol{M}^{T} \boldsymbol{B}_{f}^{T} \boldsymbol{P}-\boldsymbol{P} \boldsymbol{B}_{f} \boldsymbol{M}\right)-$ $\left(\boldsymbol{C}_{v}^{T}-\boldsymbol{P} \boldsymbol{B}_{f}\right)\left(\boldsymbol{S}+\boldsymbol{S}^{T}\right)^{-1}\left(\boldsymbol{C}_{v}^{T}-\boldsymbol{P} \boldsymbol{B}_{f}\right)^{T} \succ 0$. Pre- and postmultiplying with $\boldsymbol{P}^{-1}$ (a congruence transformation) and substitutions $\boldsymbol{X} \triangleq \boldsymbol{P}^{-1}$ and $\boldsymbol{Y} \triangleq \boldsymbol{M} \boldsymbol{P}^{-1}$ give the result $-\left(\boldsymbol{X} \boldsymbol{A}^{T}+\boldsymbol{A} \boldsymbol{X}-\boldsymbol{Y}^{T} \boldsymbol{B}_{f}^{T}-\boldsymbol{B}_{f} \boldsymbol{Y}\right)-\left(\boldsymbol{X} \boldsymbol{C}_{v}^{T}-\boldsymbol{B}_{f}\right)(\boldsymbol{S}+$ $\left.\boldsymbol{S}^{T}\right)^{-1}\left(\boldsymbol{C}_{v}^{T} \boldsymbol{X}-\boldsymbol{B}_{f}\right)^{T} \succ 0$. Applying the Schur lemma once more gives the LMI (12).

It remains to be shown that absolute stability of the unforced difference system extends to the input-to-state stability of the difference system with nonzero inputs $\boldsymbol{u}_{c}$ and $\tilde{\boldsymbol{x}}$. This follows from the fact that LMI (12) implies not only global asymptotic stability for all Lure nonlinearities in the sector, but also global exponential stability. Together with Assumptions 3 and 1 and according to [Khalil, 2002, Lemma 4.6], this implies that the forced difference system is globally ISS w.r.t. $\boldsymbol{u}_{c}$ and $\tilde{\boldsymbol{x}}$ as inputs. 
Remark 1. (Performance). The simplest way to incorporate performance goals into the design consists in ignoring the Lure nonlinearity for the purpose of performance optimization (setting it to zero). Henceforth, linear performance indices can be included in the design, based on e.g. the $H_{\infty}$ or $H_{2}$ norm, see e.g. Richter and Lunze [2009]. Optimal performance is not really achieved for the Lure system, but improvements may in practice be found over a purely stabilizing design. Absolute stability is in any case preserved by such semi-heuristic design extensions.

The stabilizing design of the Lure virtual actuator (10) is summarized in Algorithm 1.

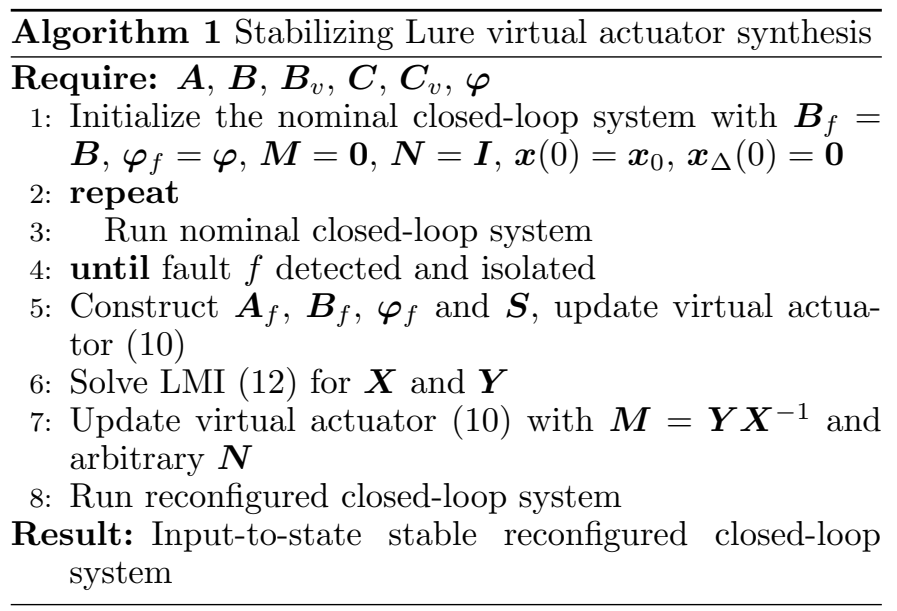

\section{SYSTEM UNDER STUDY}

To test the described method for reconfiguring stabilisers, a test setup is presented. The system in consideration is Kundur's two area system [Kundur, 1994], which exhibits different kinds of electromechanical oscillations; both local interplant and inter-area. As [Ni et al., 2000] a timedomain simulation is performed on the test system, where a PSS failure occurs. The test system and principle of reconfiguration is shown in Figure 3.
The system consists of 4 generators and 11 busses. The structure is symmetric, but with a higher load in one area, generating a power transfer from the first area to the second.

The objective is to create a reconfiguration block in case of a PSS failure as shown in Figure 3, by compensating through the other PSSs.

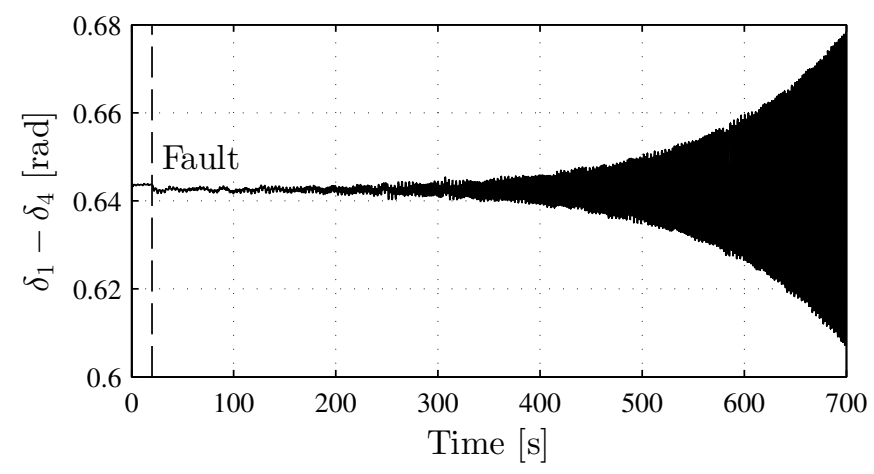

Fig. 4. The simulated response of test system after PSSG2 failure. An oscillatory and growing seperation develops in rotor angle between G1 and G4.

In the simulation case, the loadings are the same as in [Kundur, 1994], that is the generator units are loaded at $P_{m, 1}=700 \mathrm{MW}, P_{m, 2}=700 \mathrm{MW}, P_{m, 3}=719 \mathrm{MW}$ and $P_{m, 4}=700 \mathrm{MW}$. The system is stabilised by fitting PSS on all the generators. A wide-area LQ controller is used as nominal PSS (with weights $\boldsymbol{Q}=\boldsymbol{I}$ and $\boldsymbol{R}=$ $\operatorname{diag}(1,0.01,1,1)$ to intensify the role of $\mathrm{G} 2)$, to stabilise the electromechanical modes.

The reconfiguration test case is performed for a setup, where a failure of the PSS at generator 2 is introduced. The resulting time response the failure is illustrated in Figure 4. Simulation results indicate that the system becomes unstable, when a small disturbance is introduced to the generators.

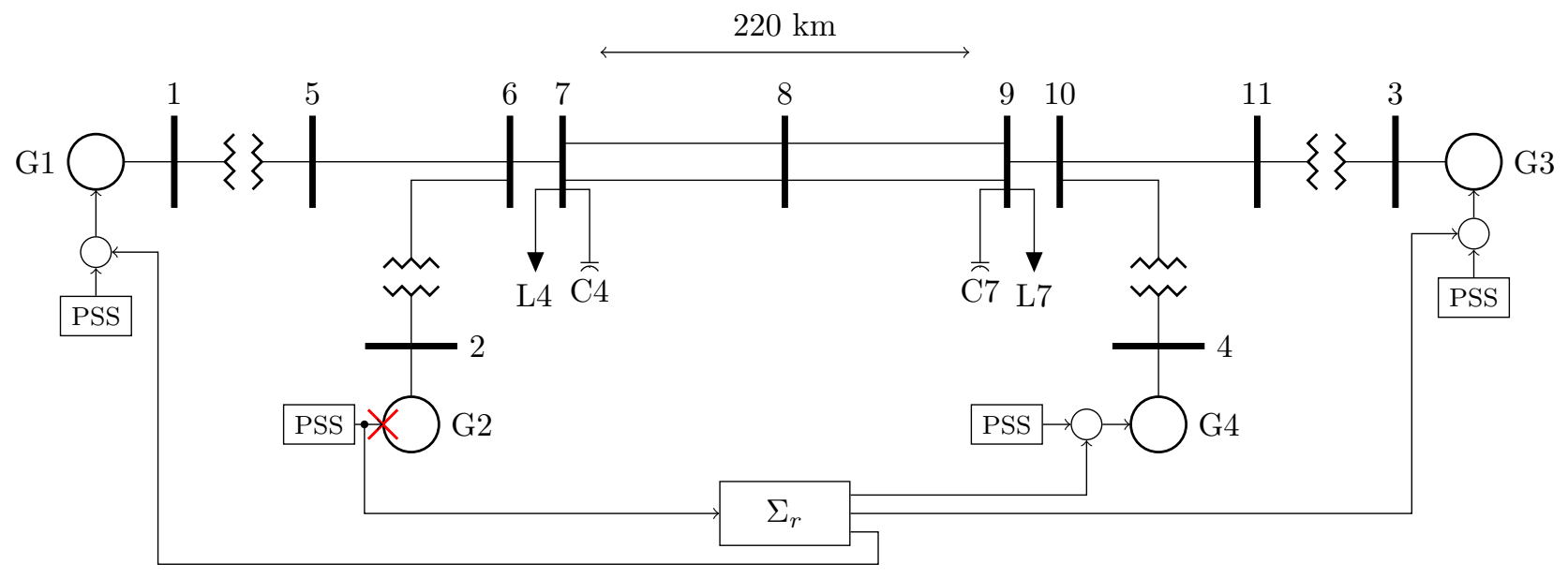

Fig. 3. Simplified illustration of the reconfiguration after a PSS failure at generator G2. The network is Kundurs two-area system. 


\section{SIMULATION RESULTS}

To test the method, a simulation of the test system is performed, with a virtual actuator calculated as in Theorem 1. A fault is introduced at $t=20 \mathrm{~s}$, where the stabiliser on G2 is set to being non-active, making the system unstable. The virtual actuator is updated according to Algorithm 1 at $t=30 \mathrm{~s}$. This result does not consider time delay due to the communication network. If the control is centralized, the nominal system would already comply with the assumptions. If not, the time delay should be considered during the reconfiguration design. The results are shown in Figure 5.
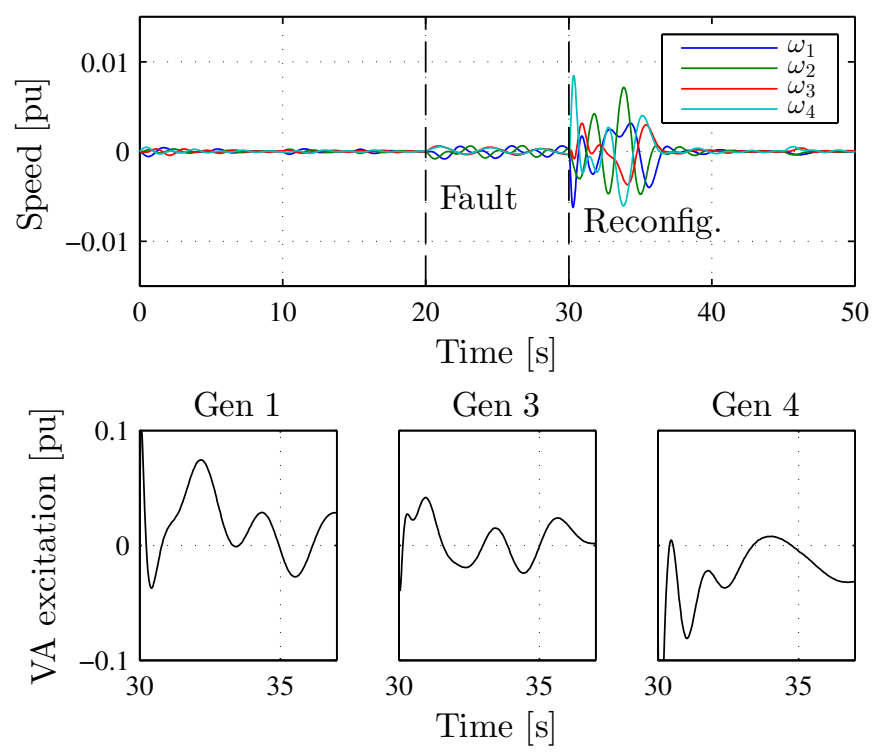

Fig. 5. The simulated response of PSS failure occuring at $t=20 \mathrm{~s}$, where a reconfiguration occurs at $t=30 \mathrm{~s}$.

A zoom of the added stabilizing signals to the healthy stabilisers are shown in Fig. 5. Compared to the nonreconfigured simulation in Fig. 4, these additional signals are able to stabilise the closed-loop system. A small switching transient is present when the virtual actuator is modified.

In the presented work, all the networks PSSs will contribute to stabilization after a failure. The method can be extended to only use nearby PSSs to stabilise the system. If the PSSs have knowledge of the control strategy of its neighbours and have wide-area state information, the additional stabilizing input can be computed locally.

\section{CONCLUSIONS}

In this work, the application of control reconfiguration after faults on power system stabilisers was tested. Using the flux-decay model an optimisation depending on system parameters, with an assumption on voltage deviations, can be performed which guarantees stability of the closed-loop system. Simulation showed its ability to stabilise the often used two-area system from [Kundur, 1994].

As the constructed reconfiguration block (the difference system) is in series with the nominal control loop, the nominal control properties are preserved. This is a salient feature of the approach, as PSSs are often designed with specific properties (such as having a washout effect on the control signal to avoid modifying the steady-state behaviour), which are preserved through the reconfiguration.

\section{REFERENCES}

M. Begovic, D. Novosel, D. Karlsson, C. Henville, and G. Michel. Wide-Area Protection and Emergency Control. Proceedings of the IEEE, 93(5):876-891, May 2005.

M. Blanke, M. Kinnaert, J. Lunze, and M. Staroswiecki. Diagnosis and Fault-Tolerant Control. Springer, 2006.

S. Boyd, L. E. Ghaouni, E. Feron, and V. Balakrishnan. Linear Matrix Inequalities in System and Control Theory. SIAM, 1994.

He Chen and Zhizhong Guo. LMI-based Wide-area Robust Damping Control. 2005 IEEE/PES Transmission \& Distribution Conference $\&$ Exposition: Asia and Pacific, pages 1-6, 2005. doi: 10.1109/TDC.2005.1546830.

He Chen, Zhizhong Guo, and Hong Bai. Wide-area Robust H2/Hinfty Control with pole placement for Damping Inter-area Oscillations. In Proc. IEEE Power Engineering Society General Meeting (PES), 2006. ISBN 1424404932.

N Kakimoto, Y. Ohsawa, and M. Hayashi. Transient Stability Analysis of Multimachine Power System with Field Flux Decays via Lyapunov's Direct Method. IEEE Transactions on Power Apparatus and Systems, PAS-99 (5):1819-1827, 1980.

I. Kamwa, R. Grondin, and Y. Hebert. Wide-area measurement based stabilizing control of large power systems-a decentralized/hierarchical approach. Power Systems, IEEE Transactions on, 16(1):136-153, 2001. ISSN 0885-8950. doi: 10.1109/59.910791.

H. K. Khalil. Nonlinear Systems. Prentice Hall, New Jersey, 3rd edition, 2002.

Prabha Kundur. Power System Stability and Control. McGraw-Hill, 1994.

Hui Ni, G.T. Heydt, and R.G. Farmer. Autonomous damping controller design for power system oscillations. In Power Engineering Society Summer Meeting, 2000. IEEE, volume 2, pages 1133-1138 vol. 2, 2000.

J. H. Richter. Reconfigurable Control of Nonlinear Dynamical Systems - a fault-hiding approach, volume 408 of LNCIS. Springer, 2011a.

J. H. Richter and J. Lunze. H-infinity-based virtual actuator synthesis for optimal trajectory recovery. In Proc. 7th IFAC Symposium on Fault Detection, Supervision and Safety of Technical Processes, Barcelona, Spain, JUL 2009. IFAC. Accepted.

Jan Richter, Maria Seron, and Jose Adrian De Dona. Virtual actuator for lure systems with lipschitz-continuous nonlinearity. In Fault Detection, Supervision and Safety of Technical Processes, volume 8, pages 222-227, 2012.

Jan H. Richter. Reconfigurable Control of Nonlinear Dynamical Systems: A Fault-hiding Approach. Springer Verlag, 2011b.

Peter W. Sauer and M. A. Pai. Power System Dynamics and Stability. Prentice Hall, 1998.

Aaron F Snyder, Nouredine Hadjsaid, Didier Georges, Lamine Mili, Arun G Phadke, Olivier Fawon, and $\mathrm{W}$ Sylvain. Inter-area oscillation damping with power system stabilizers and synchronized phasor measurements. In Proc., pages 790-794, 1998. ISBN 0780347544. 
T. Steffen. Control Reconfiguration of Dynamical Systems: Linear Approaches and Structural Tests, volume 320 of LNCIS. Springer, 2005.
Y. Zhang, G. P. Chen, O. P. Malik, and G.S. Hope. An artificial neural network based adaptive power system stabilizer. Energy Conversion, IEEE Transactions on, 8(1):71-77, 1993. 